\title{
LAMPLIGHTERS, DIESTEL-LEADER GRAPHS, RANDOM WALKS, AND HARMONIC FUNCTIONS
}

\author{
WOLFGANG WOESS
}

\begin{abstract}
The lamplighter group over $\mathbb{Z}$ is the wreath product $\mathbb{Z}_{q} \prec \mathbb{Z}$. With respect to a natural generating set, its Cayley graph is the Diestel-Leader graph $D L(q, q)$. We study harmonic functions for the "simple" Laplacian on this graph, and more generally, for a class of random walks on $D L(q, r)$, where $q, r \geq 2$. The $D L$-graphs are horocyclic products of two trees, and we give a full description of all positive harmonic functions in terms of the boundaries of these two trees. In particular, we determine the minimal Martin boundary, that is, the set of minimal positive harmonic functions.
\end{abstract}

\section{INTRODUCTION}

Think of a (typically infinite) connected graph $X$ where in each vertex there is a lamp that may be switched off (state 0 ), or switched on with $q-1$ different intensities (states $1, \ldots, q-1)$. Initially, all lamps are turned off, and a lamplighter starts at some vertex of $X$ and walks around. When he visits a vertex, he may switch the lamp sitting there into one of its $q$ different states (including "off"). Our information consists of the position $x \in X$ of the lamplighter and of the finitely supported configuration $\eta: X \rightarrow \mathbb{Z}_{q}=\{0, \ldots q-1\}$ of the lamps that are switched on, including their respective intensities. The set $\mathbb{Z}_{q}$ 々 $X$ of all such pairs $(\eta, x)$ can be equipped in several ways with a natural connected graph structure, giving rise to a lamplighter graph.

When $X$ is a Cayley graph of a group $\Gamma$ then underlying this construction, there is the wreath product $\mathbb{Z}_{q} \nmid \Gamma$, which is the semidirect product of $\Gamma$ with the group of all finitely supported functions $\eta: \Gamma \rightarrow \mathbb{Z}_{q}$ (i.e., a direct sum), on which $\Gamma$ acts by $g \eta(h)=\eta\left(g^{-1} h\right)$. Instead of $\mathbb{Z}_{q}=\mathbb{Z} /(q \mathbb{Z})$, one may of course take any other group $L$ of "lamps", leading to the wreath product $L<\Gamma$.

Various aspects of random walks on lamplighter groups have received considerable attention recently: Poisson boundary (KAImANOVICH AND VERShiK [1] and Kaimanovich [10]), rate of escape (Lyons, Pemantle and Peres [13], Erschler [8], Revelle [18]), spectral theory (GRIGORCHUK AND ŻUK 9]), and the asymptotic behaviour of transition probabilites (SAloff-Coste and Pittet [16], 17], Revelle [19]). Here, we shall consider harmonic functions.

A harmonic function on a locally finite graph is a real-valued function whose value at each vertex coincides with the arithmetic average of its values in the neighbour vertices.

Date: Version of November 27, 2003, to appear in Combinatorics, Probability \& Computing.

2000 Mathematics Subject Classification. 60J50; 05C25, 20E22, 31C05, 60G50.

Key words and phrases. lamplighter group, wreath product, Diestel-Leader graphs, trees, random walks, harmonic functions, minimal Martin boundary.

Partially supported by FWF (Austrian Science Fund) project P15577. 
More generally, we can consider the transition matrix $P$ of a random walk on the graph, suitably adapted to the graph's geometry; a harmonic function $h$ is then one that satisfies $P h=h$.

In the present paper, we shall determine all positive harmonic functions on certain Cayley graphs of the simplest lamplighter group, $\Gamma=\mathbb{Z}_{q} \imath \mathbb{Z}$. Namely, we first explain that the Diestel-Leader graph $D L(q, q)$ is a Cayley graph of $\Gamma$. More generally, if $q, r \geq 2$ then $D L(q, r)$ is obtained as a "horocyclic product" of two homogeneous trees $\mathbb{T}_{q}$ and $\mathbb{T}_{r}$ with degrees $q+1$ and $r+1$, respectively. We remark that this does not mean that $D L(q, r)$ is "almost" a tree in any sense; indeed, it is a one-ended, vertex-transitive graph which is a Cayley graph only when $r=q$. When $r \neq q$, it is believed to be an example of a transitive graph that is not quasi-isometric with any Cayley graph of some finitely generated group - see DiESTEL AND LEADER [6].

Nevertheless, we can use the boundary of each of the two trees that compose $D L(q, r)$ for giving an integral representation of all positive harmonic functions: in that representation, we start with the projections of the random walk on $D L(q, r)$ to each of the two trees and the corresponding Martin kernels. Our main result is that every positive harmonic function on $D L(q, r)$ is of the form $h=h_{1}+h_{2}$, where $h_{1}$ is obtained by lifting a harmonic function from $\mathbb{T}_{q}$ to $D L(q, r)$, and $h_{2}$ is obtained analogously from $\mathbb{T}_{r}$. Thereby, we also determine all minimal positive harmonic functions.

We now give an outline of the contents of this paper.

Section 2, although it does not contain proofs, is crucial, since it explains the geometry of the structures that we are working with, and in particular, the correspondence between lamplighter groups and Diestel-Leader graphs. As a matter of fact, it is precisely this geometric realization that allows us to determine all positive harmonic functions on $\mathbb{Z}_{q} \imath \mathbb{Z}$. At the end of 92 , we state the first main result, regarding the decomposition of positive harmonic functions over the two trees (Theorem 2.10).

In Section 3, we recall basic results on positive harmonic functions for irreducible Markov chains. In particular, we consider finite sets with boundaries, the Martin boundary at infinity and its minimal part, and the Martin compactification for nearest neighbour random walks on trees.

In Section 4, we use all the preceding ingredients to prove the Decomposition Theorem 2.10. It is then quite simple to determine all minimal positive harmonic functions (Theorem 4.4); they are the Martin kernels of the two projected random walks, up to one, resp. two exceptions. We then retranslate these results to the lamplighter group $\mathbb{Z}_{q} \prec \mathbb{Z}$ (Example 4.5).

In Section 5, we adapt the preceding results to the "switch-walk-switch" random walk, which is in some sense more natural from the point of view of the lamplighter than the simple random walk on $D L(q, q)$.

Section [6] is devoted to some additional remarks and speculations. 


\section{Diestel-LeAder GRAPHS AND LAMPlighters}

Let $\mathbb{T}=\mathbb{T}_{q}$ be the homogeneous tree with degree $q+1, q \geq 2$. A geodesic path, resp. geodesic ray, resp. infinite geodesic in $\mathbb{T}$ is a finite, resp. one-sided infinite, resp. doubly infinite sequence $\left(x_{n}\right)$ of vertices of $\mathbb{T}$ such that $d\left(x_{i}, x_{j}\right)=|i-j|$ for all $i, j$, where $d(\cdot, \cdot)$ denotes the graph distance.

Two rays are equivalent if their symmetric difference is finite. An end of $\mathbb{T}$ is an equivalence class of rays. The space of ends is denoted $\partial \mathbb{T}$, and we write $\widehat{\mathbb{T}}=\mathbb{T} \cup \partial \mathbb{T}$. For all $w, z \in \widehat{\mathbb{T}}$ there is a unique geodesic $\overline{w z}$ that connects the two. In particular, if $x \in \mathbb{T}$ and $\xi \in \partial \mathbb{T}$ then $\overline{x \xi}$ is the ray that starts at $x$ and represents $\xi$. Furthermore, if $\xi, \zeta \in \partial \mathbb{T}(\xi \neq \zeta)$ then $\overline{\zeta \xi}$ is the infinite geodesic whose two halves (split at any vertex) are rays that respresent $\zeta$ and $\xi$, respectively.

For $x, y \in \mathbb{T}, x \neq y$, we define the cone $\widehat{\mathbb{T}}(x, y)=\{w \in \widehat{\mathbb{T}}: y \in \overline{x w}\}$. The collection of all cones is the basis of a topology wich makes $\widehat{\mathbb{T}}$ a compact, totally disconnected Hausdorff space with $\mathbb{T}$ as a dense, discrete subset. We denote $\mathbb{T}(x, y)=\mathbb{T} \cap \widehat{\mathbb{T}}(x, y)$ and $\partial \mathbb{T}(x, y)=\partial \mathbb{T} \cap \mathbb{T}(x, y)$.

We fix a root $o \in \mathbb{T}$. If $w, z \in \widehat{\mathbb{T}}$, then their confluent $c=w \wedge z$ with respect to the root vertex $o$ is defined by $\overline{o w} \cap \overline{o z}=\overline{o c}$. Similarly, we choose and fix a reference end $\omega \in \partial \mathbb{T}$. For $z, v \in \widehat{\mathbb{T}} \backslash\{\omega\}$, their confluent $b=v \curlywedge z$ with respect to $\omega$ is defined by $\overline{v \omega} \cap \overline{z \omega}=\overline{b \omega}$. The Busemann function $\mathfrak{h}: \mathbb{T} \rightarrow \mathbb{Z}$ and the horocycles $H_{k}$ with respect to $\omega$ are defined as

$$
\mathfrak{h}(x)=d(x, x \curlywedge o)-d(o, x \curlywedge o) \quad \text { and } \quad H_{k}=\{x \in \mathbb{T}: \mathfrak{h}(x)=k\} .
$$

Every horocycle is infinite. Every vertex $x$ in $H_{k}$ has one neighbour $x^{-}$(its predecessor) in $H_{k-1}$ and $q$ neighbours (its successors) in $H_{k+1}$. We set $\partial^{*} \mathbb{T}=\partial \mathbb{T} \backslash\{\omega\}$.

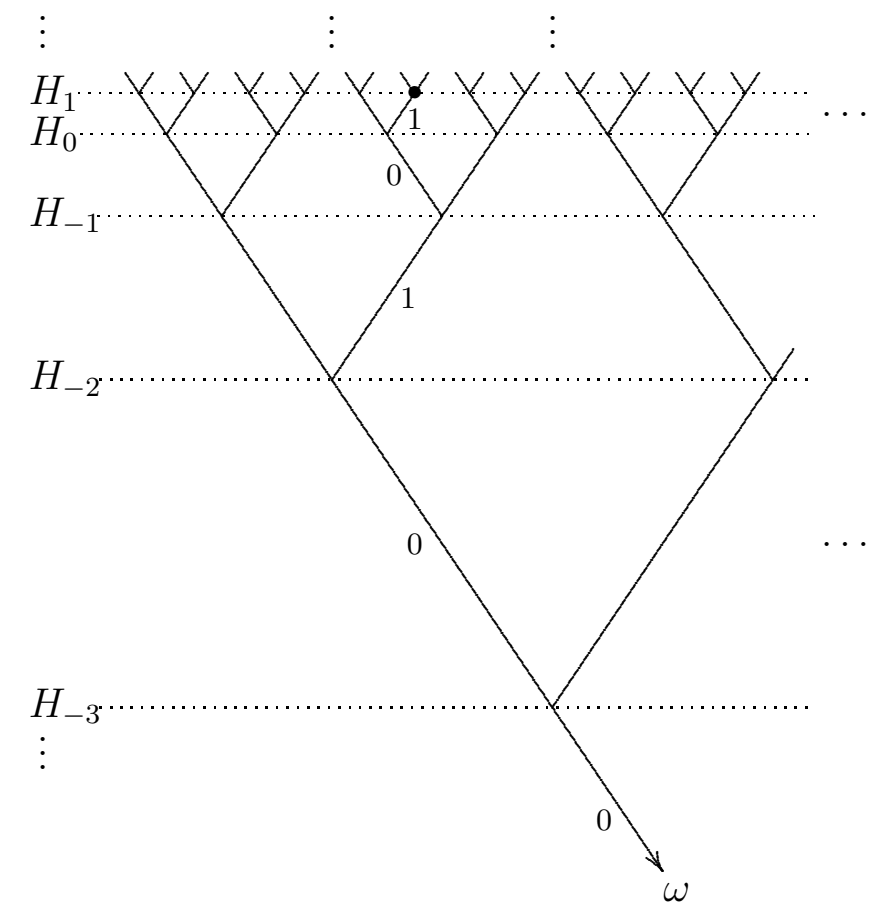

Figure 1 
(2.1) Tree and sequences. Now consider the set $\Sigma_{q}$ of all sequences $(\sigma(n))_{n \leq 0}$ over $\mathbb{Z}_{q}$ with finite support $\{n: \sigma(n) \neq 0\}$. We denote by $\tau$ the (negative) shift, $\tau \sigma(n)=\sigma(n-1)$. Then the set $\Sigma_{q} \times \mathbb{Z}$ carries the structure of $\mathbb{T}_{q}$ in horocylic layers as above: the $k$-th horocycle is $H_{k}=\Sigma_{q} \times\{k\}$, and the predecessor of vertex $x=(\sigma, k)$ is $x^{-}=(\tau \sigma, k-1)$. This corresponds to labelling the edges of $\mathbb{T}_{q}$ by elemets of $\mathbb{Z}_{q}$ such that all edges on the ray from $\omega$ to $o$ have label 0 , and for every vertex $x$ and every $\ell \in \mathbb{Z}_{q}$ there is a successor $y$ among the $q$ successors of $x$ such that the edge $[x, y]$ carries label $\ell$. See Figure 1: the origin is the leftmost point on the horocycle $H_{0}$, we have indicated the labels on the edges that lead to the point marked with a "•", and that point has coordinates $(\sigma, k)$ with $\sigma=(\ldots, 0, \ldots, 0,1,0,1)$ and $k=-1$.

Now consider two trees $\mathbb{T}_{q}$ and $\mathbb{T}_{r}$ with roots $o_{1}$ and $o_{2}$ and reference ends $\omega_{1}$ and $\omega_{2}$, respectively.

(2.2) Definition. The Diestel-Leader graph $D L(q, r)$ is

$$
D L(q, r)=\left\{x_{1} x_{2} \in \mathbb{T}_{q} \times \mathbb{T}_{r}: \mathfrak{h}\left(x_{1}\right)+\mathfrak{h}\left(x_{2}\right)=0\right\},
$$

and neighbourhood is given by

$$
x_{1} x_{2} \sim y_{1} y_{2} \Longleftrightarrow x_{1} \sim y_{1} \text { and } x_{2} \sim y_{2} .
$$

To visualize $D L(q, r)$, draw $\mathbb{T}_{q}$ in horocyclic layers with $\omega_{1}$ at the top and $\partial^{*} \mathbb{T}_{q}$ at the bottom, and right to it $\mathbb{T}_{r}$ in the same way, but upside down, with the respective horocycles $H_{k}\left(\mathbb{T}_{q}\right)$ and $H_{-k}\left(\mathbb{T}_{r}\right)$ on the same level. Connect the two origins $o_{1}, o_{2}$ by an elastic spring. It is allowed to move along each of the two trees, may expand infinitely, but must always remain in horizontal position. The vertex set of $D L_{q, r}$ consists of all admissible positions of the spring. From a position $x_{1} x_{2}$ with $\mathfrak{h}\left(x_{1}\right)+\mathfrak{h}\left(x_{2}\right)=0$ the spring may move downwards to one of the $r$ successors of $x_{2}$ in $\mathbb{T}_{r}$, and at the same time to the predecessor of $x_{1}$ in $\mathbb{T}_{q}$, or it may move upwards in the analogous way. Such a move corresponds to going to a neighbour of $x_{1} x_{2}$. Figure 2 depicts $D L(2,2)$.

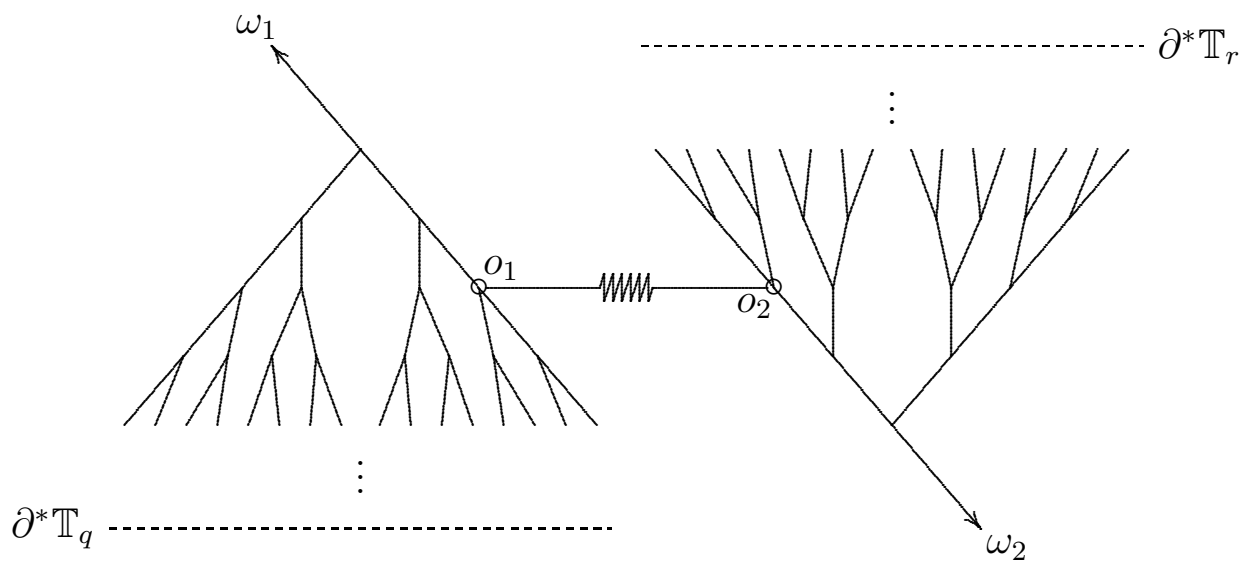

Figure 2 
As the reference point in $D L(q, r)$, we choose $o=o_{1} o_{2}$. We shall keep in mind that $\mathbb{T}_{q}$ is the first and $\mathbb{T}_{r}$ the second tree; when $r=q$, it will be sometimes convenient to write $\mathbb{T}^{1}$ and $\mathbb{T}^{2}$ for the first and second trees, both copies of $\mathbb{T}_{q}$.

Next, we explain what the lamplighter group $\mathbb{Z}_{q} \prec \mathbb{Z}$ has to do with $D L(q, q)$. Let $(\eta, k) \in \mathbb{Z}_{q} \prec \mathbb{Z}$, and recall that $\eta: \mathbb{Z} \rightarrow \mathbb{Z}_{q}$ is a finitely supported configuration. We identify $(\eta, k)$ with the vertex $x_{1} x_{2} \in D L(q, q)$, where according to (2.1), the vertices $x_{i}$ are given by

$$
\begin{gathered}
x_{1}=\left(\eta_{k}^{-}, k\right) \quad \text { and } \quad x_{2}=\left(\eta_{k}^{+},-k\right), \quad \text { where } \\
\eta_{k}^{-}=(\eta(k+n))_{n \leq 0} \text { and } \eta_{k}^{+}=(\eta(k+1-n))_{n \leq 0},
\end{gathered}
$$

that is, we split $\eta$ at $k$, with $\eta_{k}^{-}=\left.\eta\right|_{(-\infty, k]}$ and $\eta_{k}^{+}=\left.\eta\right|_{[k+1, \infty)}$, both written as sequences over the non-positive integers.

This is clearly a one-to-one correspondence between $D L(q, q)$ and $\mathbb{Z}_{q} \prec \mathbb{Z}$, and it is also straighforward that this group acts transitively and fixed-point-freely on the graph: the action of $m \in \mathbb{Z}$ is given by $x_{1} x_{2}=\left(\sigma_{1}, k\right)\left(\sigma_{2},-k\right) \mapsto y_{1} y_{2}=\left(\sigma_{1}, k+m\right)\left(\sigma_{2},-k+m\right)$, and the action of the group of configurations is pointwise addition modulo $q$ in the obvious way; the reader is invited to work out the simple details. We have to determine the symmetric set of generators of our group with respect to which $D L(q, q)$ is its Cayley graph. (Here, we mean the right Cayley graph, where an edge corresponds to multiplying with a generator on the right.)

Stepping from a vertex $x_{1} x_{2}$ to $y_{1} x_{2}^{-}$, where $y_{1}$ is one of the successors of $x_{1} \in H_{k}\left(\mathbb{T}^{1}\right)$ (horocycle in the first tree) means that the lamplighter walks from position $k$ to $k+1$ and then switches the lamp at the new position to some state in $\mathbb{Z}_{q}$. Thus, the "downward" edges of this type correspond to multiplying on the right with the group elements $\left(\delta_{1}^{\ell}, 1\right)$, $\ell \in \mathbb{Z}_{q}$, where $\delta_{k}^{\ell}$ is the configuration with value $\ell$ at $k$ and 0 elsewhere. On the other hand, we have the "upward" edges from $x_{1} x_{2}$ to $x_{1}^{-} y_{2}$, where $y_{2}$ is one of the successors of $x_{2} \in H_{-k}\left(\mathbb{T}^{2}\right)$ (horocycle in the second tree). They correspond to multiplying on the right with the inverses of the above generators, i.e., the elements $\left(\delta_{0}^{\ell},-1\right)$, where $\ell \in \mathbb{Z}_{q}$.

Thus the simple random walk on $D L(q, q)$ is the following lamplighter walk: its law, the probability measure on $\mathbb{Z}_{q} \prec \mathbb{Z}$ that describes the one step transition probabilites, is equidistribution on

$$
\left\{\left(\delta_{1}^{\ell}, 1\right),\left(\delta_{0}^{\ell},-1\right): \ell \in \mathbb{Z}_{q}\right\}
$$

If at some step, the lamplighter stands at $k \in \mathbb{Z}$, (s)he chooses with equal probability either to step to $k+1$ and then to switch the lamp at $k+1$ to a random state, or (s)he chooses to switch the lamp at $k$ to a random state (before leaving $k$ ) and then to step to $k-1$. While this is a symmetric random walk on $\mathbb{Z}_{q} \prec \mathbb{Z}$, resp. $D L(q, q)$, this type of action does not appear "symmetric" from the point of view of the lamplighter. For this reason, other types of "simple" random walks have been considered in the past: the one whose law is equidistribution on

$$
\left\{(\mathbf{0}, \pm 1),\left(\delta_{0}^{\ell}, 0\right): 0 \neq \ell \in \mathbb{Z}_{q}\right\}
$$


("walk or switch"), and the one where the lamplighter standing at $k$ first switches the lamp where he stands to a random state, then walks to $k \pm 1$, and then switches the lamp at the arrival point to a random state ("switch-walk-switch"). The corresponding law is equidistribution on

$$
\left\{\left(\delta_{0}^{\ell}+\delta_{ \pm 1}^{m}, \pm 1\right): \ell, m \in \mathbb{Z}_{q}\right\}
$$

Harmonic functions for the "walk or switch" model cannot be determined by the methods that we elaborate here, since it is not very well adapted to the structure of $D L(q, q)$, see the comments at the end.

On the other hand, the "switch-walk-switch" model (2.6) corresponds to simple random walk on the following modification of $D L(q, q)$ : in the first of the two trees, we add edges between every vertex and the siblings of its predecessor (i.e., its "uncles"), and the resulting neighbourhood relation on the horocyclic product is as in Definition 2.2. It will be easy to adapt our results to this random walk.

In the first place, we shall study the following slight generalization $P=P_{\alpha}$ of simple random walk on $D L(q, r)$, where $0<\alpha<1$. For $x_{1} x_{2} \in D L(q, r)$

$$
p\left(x_{1} x_{2}, y_{1} y_{2}\right)= \begin{cases}\alpha / q & \text { if } y_{1}^{-}=x_{1} \text { and } y_{2}=x_{2}^{-} \\ (1-\alpha) / r & \text { if } y_{1}=x_{1}^{-} \text {and } y_{2}^{-}=x_{2} \\ 0 & \text { otherwise. }\end{cases}
$$

$P$ acts on functions $h: D L(q, r) \rightarrow \mathbb{R}$ by

$$
P h\left(x_{1} x_{2}\right)=\sum_{y_{1} y_{2}} p\left(x_{1} x_{2}, y_{1} y_{2}\right) h\left(x_{1} x_{2}\right)
$$

A harmonic, or more precisely, $P_{\alpha}$-harmonic function, is one that satisfies $P h=h$.

We can consider the projections $P_{1}=P_{1, \alpha}$ and $P_{2}=P_{2,1-\alpha}$ of $P_{\alpha}$ on $\mathbb{T}_{q}$ and $\mathbb{T}_{r}$, respectively:

$$
p_{1}\left(x_{1}, y_{1}\right)=\left\{\begin{array}{ll}
\alpha / q & \text { if } y_{1}^{-}=x_{1} \\
(1-\alpha) & \text { if } y_{1}=x_{1}^{-} \\
0 & \text { otherwise }
\end{array} \quad p_{2}\left(x_{1}, y_{2}\right)= \begin{cases}\alpha & \text { if } y_{2}=x_{2}^{-} \\
(1-\alpha) / r & \text { if } y_{2}^{-}=x_{2} \\
0 & \text { otherwise }\end{cases}\right.
$$

The following is straightforward.

(2.9) Lemma. (a) If $h_{1}$ is a $P_{1}$-harmonic function on $\mathbb{T}_{q}$, then $h\left(x_{1} x_{2}\right)=h_{1}\left(x_{1}\right)$, $x_{1} x_{2} \in D L(q, r)$, defines a P-harmonic function on $D L(q, r)$.

(b) If $h_{2}$ is a $P_{2}$-harmonic function on $\mathbb{T}_{q}$, then $h\left(x_{1} x_{2}\right)=h_{2}\left(x_{2}\right), x_{1} x_{2} \in D L(q, r)$, defines a P-harmonic function on $\operatorname{DL}(q, r)$.

Our first main result is the following.

(2.10) Theorem. If $h$ is a non-negative P-harmonic function on $D L(q, r)$, then there are non-negative $P_{i}$-harmonic functions $h_{i}, i=1,2$, on $\mathbb{T}_{q}$ and $\mathbb{T}_{r}$, respectively, such that

$$
h\left(x_{1} x_{2}\right)=h_{1}\left(x_{1}\right)+h_{2}\left(x_{2}\right) \quad \text { for all } x_{1} x_{2} \in D L(q, r)
$$


Conversely, it is of course clear that every sum of the latter form defines a $P$-harmonic function. Recall that in this type of decomposition, $x_{2}$ cannot vary independently of $x_{1}$, since one must have $\mathfrak{h}\left(x_{1}\right)+\mathfrak{h}\left(x_{2}\right)=0$.

The next short section contains some basic preparatory material for the proof of Theorem 2.10 ,

\section{BASIC RESUltS ABOUT HARMONIC FUNCTIONS}

Let $X$ be a denumerable set and $P=(p(x, y))_{x, y \in X}$ the stochastic transition matrix of a Markov chain $\left(Z_{n}\right)_{n \geq 0}$ on $X$. We write $\operatorname{Pr}_{x}$ for probability conditioned to the starting point $Z_{0}=x$. The $n$-step transition probability $p^{(n)}(x, y)=\operatorname{Pr}_{x}\left[Z_{n}=y\right]$ is the $(x, y)$-entry of the matrix power $P^{n}$. We assume that $P$ is irreducible: $\forall x, y \exists n: p^{(n)}(x, y)>0$.

As above, a function $h$ on $X$ is called P-harmonic or just harmonic at $x$, if $P h(x)=$ $h(x)$, where $P h(x)=\sum_{y} p(x, y) h(y)$. It is called harmonic when it is harmonic at each $x$.

For a subset $A \subset X$, we define the stopping time

$$
s^{A}=\inf \left\{n \geq 0: Z_{n} \in A\right\} .
$$

For $y \in X$, we write $s^{y}=s^{\{y\}}$. Given $x, y \in X$, let

$$
F(x, y)=\operatorname{Pr}_{x}\left[s^{y}<\infty\right] \text { and } F^{A}(x, y)=\operatorname{Pr}_{x}\left[s^{y} \leq s^{A}, s^{y}<\infty\right]
$$

Thus, $F(x, y)$ is the probability to ever reach $y$, starting from $x$. The function $F(\cdot, y)$ is harmonic in $X \backslash\{y\}$. Furthermore, if $y \in A$, then $F^{A}(\cdot, y)$ is harmonic in $X \backslash A$.

(3.2) Harmonic functions on finite sets. Let $S$ be a finite subset of $X$. Define its boundary and interior by

$$
\partial S=\{y \in S: p(y, X \backslash S)>0\} \quad \text { and } \quad S^{o}=S \backslash \partial S .
$$

For the sake of simplicity, we assume that the restriction of $P$ to $S^{o}$ is irreducible. We define

$$
\mathcal{H}(P, S)=\left\{h: S \rightarrow \mathbb{R} \mid h \text { is harmonic in } S^{o}\right\}
$$

The following is very well known.

(3.3) Proposition. Under the above assumptions, the functions $F^{\partial S}(\cdot, y), y \in \partial S$, constitute a basis of the linear space $\mathcal{H}(P, S)$. Every $h \in \mathcal{H}(P, S)$ is uniquely respresented as

$$
h=\sum_{y \in \partial S} F^{\partial S}(\cdot, y) h(y)
$$

Proof. The functions $F^{\partial S}(\cdot, y), y \in \partial S$, are linearly independent, since $F^{\partial S}(x, y)=\delta_{x}(y)$ for $x, y \in \partial S$. Given $h \in \mathcal{H}(P, S)$, let $g=\sum_{y \in \partial S} F^{\partial S}(\cdot, y) h(y)$. Then $g \in \mathcal{H}(P, S)$, and $\left.(g-h)\right|_{\partial S} \equiv 0$. By the Minimum Principle, every function in $\mathcal{H}(P, S)$ attains its minimum (and its maximum) on the boundary. Therefore $g=h$ on $S$.

(3.4) Positive and minimal harmonic functions. Regarding the following material, see WoEss [20], §24 for a more detailed outline and many references. 
We return to the infinite set $X$ with irreducible transition matrix $P$. For the sake of simplicity, we assume that $P$ has finite range, i.e., $\{y: p(x, y)>0\}$ is finite for all $x \in X$. The set $\mathcal{H}^{+}=\mathcal{H}^{+}(P, X)$ of non-negative $P$-harmonic functions constitutes a convex cone that is closed in the topology of pointwise convergence. We choose a reference point $o \in X$. Then the set $\mathcal{B}=\left\{h \in \mathcal{H}^{+}: h(o)=1\right\}$ is a compact, convex base of the cone $\mathcal{H}^{+}$. Its extremal elements are called minimal harmonic functions. Thus, $h \in \mathcal{H}^{+}$is minimal if

$$
h(o)=1 \quad \text { and } \quad h \geq h_{1} \in \mathcal{H}^{+} \Longrightarrow h_{1} / h \equiv \text { constant. }
$$

The set $\mathcal{B}_{\text {min }}$ of minimal harmonic functions is a Borel subset of $\mathcal{B}$, and every $h \in \mathcal{H}^{+}$is an integral of minimal ones with respect to a Borel measure on $\mathcal{B}_{\text {min }}$. This can be made more precise by the following construction. Define the Martin kernel

$$
K(x, y)=F(x, y) / F(o, y) .
$$

The Martin compactification is the smallest metrizable compactification of $X$ containing $X$ as a discrete, dense subset, and to which all functions $K(x, \cdot), x \in X$, extend continuously. The Martin boundary $\mathcal{M}=\mathcal{M}(P)$ is the ideal boundary added to $X$ in this compactification. Then every minimal harmonic function is of the form $K(\cdot, \xi)$ for some $\xi \in \mathcal{M}$, and the set

$$
\mathcal{M}_{\text {min }}=\{\xi \in \mathcal{M}: K(\cdot, \xi) \text { is minimal harmonic }\}
$$

is a Borel set. The Poisson-Martin Representation Theorem says that for every $h \in \mathcal{H}^{+}$ there is a unique Borel measure $\nu^{h}$ on $\mathcal{M}$ with $\nu^{h}\left(\mathcal{M} \backslash \mathcal{M}_{\text {min }}\right)=0$ such that

$$
h(x)=\int_{\mathcal{M}} K(x, \cdot) d \nu^{h} \quad \forall x \in X .
$$

Furthermore, considering the constant harmonic function $\mathbf{1}$, we set $\nu=\nu^{\mathbf{1}}$. Then every bounded harmonic function $h$ has a unique representation as above, where $d \nu^{h}(\xi)=$ $\varphi(\xi) d \nu(\xi)$ with $\varphi \in L^{\infty}(\mathcal{M}, \nu)$. The probability space $(\mathcal{M}, \nu)$ is a model of the Poisson boundary of the random walk. While the Martin boundary is a topological object, the Poisson boundary is a measure theoretical one, and finding it means to determine it up to isomorphisms between measure spaces.

See Kaimanovich AnD VERSHIK [1] for a profound introduction and impressive results regarding Poisson boundaries of random walks on groups, KAIMANOviCH [10] for lamplighter groups over $\mathbb{Z}^{d}$ and other semidirect products, and KAIMANOVICH AND WoEss [12] for Poisson boundaries of random walks on homogenenous graphs, including the Diestel-Leader graphs.

(3.5) Harmonic functions on trees. Next, let us suppose that $X=T$ carries the structure of an infinite, locally finite tree. We assume that $P$ is of nearest neighbour type, i.e.,

$$
p(x, y)>0 \Longleftrightarrow x \sim y \text { in } T .
$$

( $\sim$ denotes neighbourhood.) We also assume that the random walk (Markov chain) with transition matrix $P$ is transient, that is, $\sum_{n} p^{(n)}(x, y)<\infty$ for some $(\Longleftrightarrow$ all $) x, y \in T$. Geodesics and boundary of $T$ are defined as in $\$ 2$, with the general tree $T$ in the place 
of $\mathbb{T}_{q}$. The results regarding the Martin compactification in this setting are contained in the seminal paper by CARTIER [4].

The basic link between tree structure and random walk is the following well-known lemma, see e.g. Cartier [4], or Woess [20, Lemmas 1.23 and 1.13(d).

(3.7) Lemma. For a nearest neighbour random walk on a tree $T$,

$$
F(x, y)=F(x, w) F(w, z) \quad \text { for all } x, y \in T \text { and } w \in \overline{x y}
$$

Furthermore, if $x \sim y$, then

$$
F(y, x)=p(y, x)+\sum_{w \neq x} p(y, w) F(w, y) F(y, x) .
$$

For $x, y \in T$, let $c=x \wedge y$ be their confluent with respect to $o$. Then the lemma implies that $K(x, y)=K(x, c)$. From here, the following is almost immediate.

(3.8) Proposition. Suppose that $P$ defines a transient nearest neighbour random walk on the locally finite tree $T$ with root o. Then the Martin compactification is the end compactification $\widehat{T}$, and for $\xi \in \partial T$, the Martin kernel is given by

$$
K(x, \xi)=K(x, c)=\frac{F(x, c)}{F(o, c)}, \quad \text { where } \quad c=x \wedge \xi
$$

Furthermore, each function $K(\cdot, \xi), \xi \in \partial T$, is minimal harmonic.

For various different proofs, see Cartier [4, Picardello, Taibleson and Woess [15], or WoEss [20], §26, or also the one which is implicit in the proof of Theorem [2.10] below.

(3.9) Example. Consider the random walk on $\mathbb{T}_{q}$ with transition matrix $P_{1}=P_{1, \alpha}$, defined in 2.8. It is clear that for this random walk, the probabilities

$$
F_{1}^{-}=F_{1}\left(x, x^{-}\right) \quad \text { and } \quad F_{1}^{+}=F_{1}\left(x^{-}, x\right)
$$

are independent of $x \in \mathbb{T}_{q}\left(x^{-}\right.$is the predecessor with respect to $\left.\omega\right)$. Using Lemma 3.7. we find the two quadratic equations

$$
F_{1}^{-}=(1-\alpha)+\alpha\left(F_{1}^{-}\right)^{2} \quad \text { and } \quad F_{1}^{+}=\frac{\alpha}{q}+(q-1) \frac{\alpha}{q} F_{1}^{-} F_{1}^{+}+(1-\alpha)\left(F_{1}^{+}\right)^{2} .
$$

Among the two solutions of each equation, the smaller one is the right one (compare e.g. with the generating functions argument in the proof of Lemma 1.24 in WoEss [20]). Thus

$$
F_{1}^{-}=\left\{\begin{array}{ll}
\frac{1-\alpha}{\alpha} & \text { if } \alpha \geq \frac{1}{2}, \\
1 & \text { if } \alpha \leq \frac{1}{2},
\end{array} \quad F_{1}^{+}= \begin{cases}\frac{1}{q} & \text { if } \alpha \geq \frac{1}{2} \\
\frac{\alpha}{(1-\alpha) q} & \text { if } \alpha \leq \frac{1}{2}\end{cases}\right.
$$


We can now compute the associated Martin kernels $K_{1}(\cdot, \xi), \xi \in \partial \mathbb{T}_{q}$. First, since $x \wedge \omega=$ $x \curlywedge o$ (where $\wedge$ and $\curlywedge$ denote confluents with respect to $o$ and $\omega$ ), it is immediate that

$$
K_{1}(x, \omega)=\left(F_{1}^{-}\right)^{\mathfrak{h}(x)}= \begin{cases}\left(\frac{1-\alpha}{\alpha}\right)^{\mathfrak{h}(x)} & \text { if } \alpha \geq \frac{1}{2} \\ 1 & \text { if } \alpha \leq \frac{1}{2}\end{cases}
$$

Next, if $\xi \in \partial^{*} \mathbb{T}_{q}$, we set $c=x \wedge \xi$ and write $k=d(o, o \curlywedge x), l=d(x, o \curlywedge x)$, so that $\mathfrak{h}(x)=l-k$. We distinguish two cases, see Figure 3 .
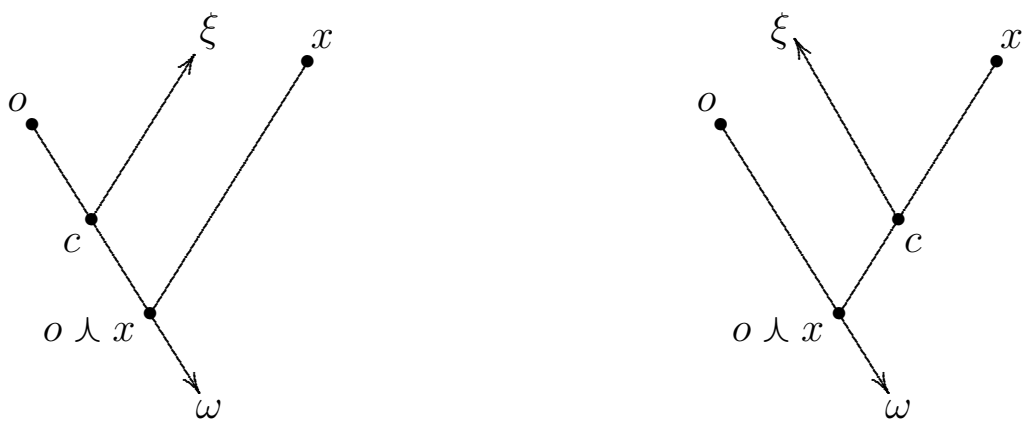

Figure 3

Case 1. $c$ lies between $o$ and $o \curlywedge x$. Let $s=d(o, c)$. Then

$$
K_{1}(x, \xi)=\frac{\left(F_{1}^{-}\right)^{l}\left(F_{1}^{+}\right)^{k-s}}{\left(F_{1}^{-}\right)^{s}}=K_{1}(x, \omega)\left(F_{1}^{-} F_{1}^{+}\right)^{k-s}=K_{1}(x, \omega)\left(F_{1}^{-} F_{1}^{+}\right)^{\mathfrak{h}(o \curlywedge \xi)-\mathfrak{h}(x \curlywedge \xi)}
$$

Case 2. $c$ lies between $o \curlywedge x$ and $x$. Let $r=d(x, c)$. Then

$$
K_{1}(x, \xi)=\frac{\left(F_{1}^{-}\right)^{r}}{\left(F_{1}^{-}\right)^{k}\left(F_{1}^{+}\right)^{l-r}}=K_{1}(x, \omega)\left(F_{1}^{-} F_{1}^{+}\right)^{r-l}=K_{1}(x, \omega)\left(F_{1}^{-} F_{1}^{+}\right)^{\mathfrak{h}(o \curlywedge \xi)-\mathfrak{h}(x \curlywedge \xi)}
$$

We write $\mathfrak{h}(x, \xi)=d(x, c)-d(o, c)$, the horocycle number with respect to $\xi$, while $\mathfrak{h}(x)=$ $\mathfrak{h}(x, \omega)$. Also, we set $\rho=\left(F_{1}^{-} F_{1}^{+}\right)^{1 / 2}$. Then we find in both cases

$$
K_{1}(x, \xi)=K_{1}(x, \omega) \rho^{\mathfrak{h}(x, \xi)-\mathfrak{h}(x)}, \quad \text { where } \quad \rho=\min \left\{\frac{1-\alpha}{\alpha q}, \frac{\alpha}{(1-\alpha) q}\right\}^{1 / 2} .
$$

In particular, if $\alpha=1 / 2$ then

$$
K_{1}(x, \omega)=1 \quad \text { and } \quad K_{1}(x, \xi)=q^{(\mathfrak{h}(x)-\mathfrak{h}(x, \xi)) / 2} \quad \text { for } \quad \xi \in \partial^{*} \mathbb{T}_{q} .
$$

\section{Minimal harmonic Functions on $D L(q, r)$}

After all these preliminaries, the proof of Theorem 2.10 depends in the first place on the way how we look at the underlying structure.

Proof of Theorem 2.10. In $D L(q, r)$, consider the subgraph spanned by all vertices $x_{1} x_{2}$ with $-n \leq \mathfrak{h}\left(x_{1}\right) \leq n$. It is not connected. We denote by $S=S^{(n)}$ the connected component of the root $O_{1} O_{2}$. 
Let $a_{1}=a_{1}^{(n)} \in \mathbb{T}_{q}$ be the vertex on $\overline{o_{1} \omega_{1}}$ at distance $d\left(a_{1}, o_{1}\right)=n$. Then $a_{1}$ can be viewed as the root of the $q$-ary rooted tree $S_{1}=S_{1}^{(n)}=\left\{x_{1} \in \mathbb{T}_{q}:-n \leq \mathfrak{h}\left(x_{1}\right) \leq\right.$ $\left.n, a_{1} \in \overline{x_{1} \omega_{1}}\right\}$ of height $2 n$, whose set of leaves (elements with $\mathfrak{h}\left(x_{1}\right)=n$ ) is denoted $\partial^{*} S_{1}$. Analogously, we define $a_{2}=a_{2}^{(n)}$, the $r$-ary rooted tree $S_{2}=S_{2}^{(n)}$, and it set of leaves $\partial^{*} S_{2}$. Then

$$
S=\left\{x_{1} x_{2} \in S_{1} \times S_{2}: \mathfrak{h}\left(x_{1}\right)+\mathfrak{h}\left(x_{2}\right)=0\right\}
$$

is the horocyclic product of $S_{1}$ and $S_{2}$, see Figure 4 .

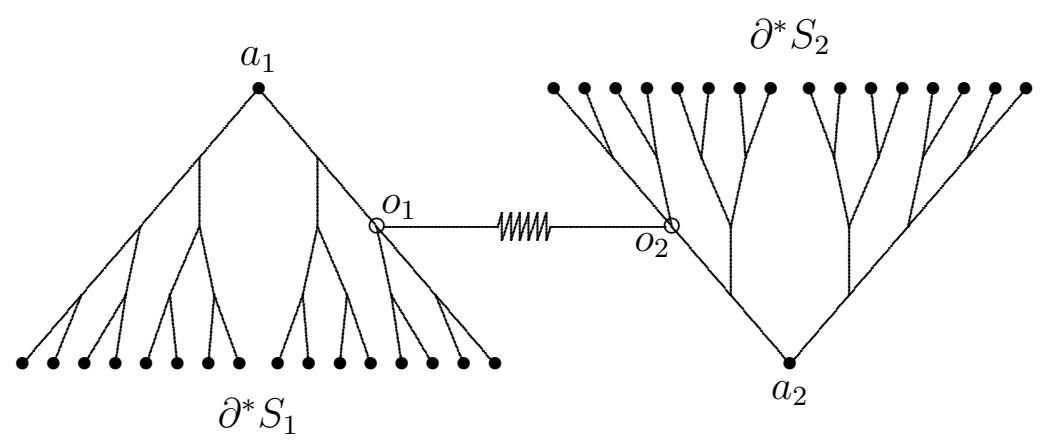

Figure 4

One may imagine $S$ as a tetrahedron. Two of its faces are copies of $S_{1}$ that meet at the common bottom edge $\partial^{*} S_{1} \times\left\{a_{2}\right\}$, and the other two faces are copies of $S_{2}$ that meet at the common top edge $\left\{a_{1}\right\} \times \partial^{*} S_{2}$. The boundary of $S_{i}$ is $\left\{a_{i}\right\} \cup \partial^{*} S_{i}$.

We now restrict $P$ to $S$, and also the projections $P_{1}$ to $S_{1}$ and $P_{2}$ to $S_{2}$. Then the boundary of $S$ in the sense of (3.2) is

$$
\partial S=\left(\partial^{*} S_{1} \times\left\{a_{2}\right\}\right) \cup\left(\left\{a_{1}\right\} \times \partial^{*} S_{2}\right) .
$$

As in Lemma 2.9] if $h_{i} \in \mathcal{H}\left(P_{i}, S_{i}\right)$, then it lifts to a function in $\mathcal{H}(P, S)$.

In particular, if $y_{1} \in \partial^{*} S_{1}$ then $h\left(x_{1} x_{2}\right)=F_{1}^{\partial S_{1}}\left(x_{1}, y_{1}\right)$ defines a function in $\mathcal{H}(P, S)$ with value one at $y_{1} a_{2}$ and value 0 in $\partial S \backslash\left\{y_{1} a_{2}\right\}$. But, by Proposition 3.3. these properties characterize the function $x_{1} x_{2} \mapsto F^{\partial S}\left(x_{1} x_{2}, y_{1} a_{2}\right)$ on $S$. Therefore, for all $x_{1} x_{2} \in S$,

$$
\begin{array}{ll}
F^{\partial S}\left(x_{1} x_{2}, y_{1} a_{2}\right)=F_{1}^{\partial S_{1}}\left(x_{1}, y_{1}\right) & \forall y_{1} \in \partial^{*} S_{1} \quad \text { and } \\
F^{\partial S}\left(x_{1} x_{2}, a_{1} y_{2}\right)=F_{2}^{\partial S_{2}}\left(x_{2}, y_{2}\right) & \forall y_{2} \in \partial^{*} S_{2}
\end{array}
$$

Applying Proposition 3.3 once more, we see that every $h \in \mathcal{H}(P, S)$ can be written uniquely as

$$
\begin{aligned}
h\left(x_{1} x_{2}\right) & =h_{1}\left(x_{1}\right)+h_{2}\left(x_{2}\right) \quad \forall x_{1} x_{2} \in S, \quad \text { where } \\
h_{1}\left(x_{1}\right) & =\sum_{y_{1} \in \partial^{*} S_{1}} F_{1}^{\partial S_{1}}\left(x_{1}, y_{1}\right) h\left(y_{1} a_{2}\right) \quad \text { and } \\
h_{2}\left(x_{2}\right) & =\sum_{y_{2} \in \partial^{*} S_{2}} F_{2}^{\partial S_{2}}\left(x_{2}, y_{2}\right) h\left(a_{1} y_{2}\right) .
\end{aligned}
$$

This is true, in particular, if $h$ is $P$-harmonic on the whole of $D L(q, r)$, since its restriction to $S=S^{(n)}$ is in $\mathcal{H}(P, S)$. Furthermore, if $h$ is non-negative then so are $h_{1}$ and $h_{2}$. Note, 
however, that $h_{i}=h_{i}^{(n)}(i=1,2)$ depend on $n$, and it is by no means true that the restriction of $h_{i}^{(n+1)}$ to $S_{i}^{(n)}$ might coincide with $h_{i}^{(n)}$. We have to study the behaviour of $h_{i}^{(n)}$ when $n \rightarrow \infty$, and this is the point where the assumption of non-negativity of $h$ will be used. We define

$$
K_{i}^{(n)}\left(x_{i}, y_{i}\right)=\frac{F_{i}^{\partial S_{i}}\left(x_{i}, y_{i}\right)}{F_{i}^{\partial S_{i}}\left(o_{i}, y_{i}\right)}, x_{i} \in S_{i}, y_{i} \in \partial S_{i}, S_{i}=S_{i}^{(n)}, i=1,2 .
$$

Then we can rewrite the functions $h_{i}$ of (4.2) as

$$
\begin{gathered}
h_{i}\left(x_{i}\right)=\sum_{y_{i} \in \partial S_{i}} K_{i}^{(n)}\left(x_{i}, y_{i}\right) \lambda_{i}\left(y_{i}\right), \quad \text { where } \\
\lambda_{1}\left(a_{1}\right)=0, \quad \lambda_{1}\left(y_{1}\right)=h\left(y_{1} a_{2}\right) / F_{1}^{\partial S_{1}}\left(o_{1}, y_{1}\right), y_{1} \in \partial^{*} S_{1}, \\
\lambda_{2}\left(a_{2}\right)=0, \quad \lambda_{2}\left(y_{2}\right)=h\left(a_{1} y_{2}\right) / F_{2}^{\partial S_{2}}\left(o_{2}, y_{2}\right), y_{2} \in \partial^{*} S_{2} .
\end{gathered}
$$

Of course, also $\lambda_{i}\left(y_{i}\right)=\lambda_{i}^{(n)}\left(y_{i}\right)$ depends on $n$. For $\xi_{1} \in \partial \mathbb{T}_{q}$, we define

$$
K_{1}^{(n)}\left(x_{1}, \xi_{1}\right)=K_{1}^{(n)}\left(x_{1}, y_{1}\right), \quad \text { if } \xi_{1} \in \mathbb{T}_{q}\left(o_{1}, y_{1}\right) \text { with } y_{1} \in \partial S_{1}
$$

Then $K_{1}^{(n)}\left(x_{1}, \cdot\right)$ is locally constant (whence continuous) on $\partial \mathbb{T}_{q}$. (Recall that $\partial \mathbb{T}_{q}$ is compact and totally disconnected.) We define a non-negative Borel-measure $\nu_{1}^{(n)}$ on $\partial \mathbb{T}_{q}$ by

$$
\begin{aligned}
& \nu_{1}^{(n)}\left(\partial \mathbb{T}_{q}\left(o_{1}, a_{1}^{-}\right)\right)=0 \text { and } \\
& \nu_{1}^{(n)}\left(\partial \mathbb{T}_{q}\left(o_{1}, w_{1}\right)\right)=\lambda_{1}^{(n)}\left(y_{1}\right) q^{\mathfrak{h}\left(w_{1}\right)-n} \quad \text { if } w_{1} \in \mathbb{T}_{q}\left(o_{1}, y_{1}\right), y_{1} \in \partial^{*} S_{1}^{(n)} .
\end{aligned}
$$

This defines a finitely additive, non-negative measure on the semiring of all sets $\partial \mathbb{T}_{q}\left(o_{1}, x_{1}\right)$, $x_{1} \in \mathbb{T}_{q} \backslash\left\{o_{1}\right\}$. Since all these sets are open and compact, the measure is sigma-additive on that semiring and extends to a unique non-negative Borel measure on $\partial \mathbb{T}_{q}$. We proceed in precisely the same way on the second tree, and also get a non-negative Borel measure $\nu_{2}^{(n)}$ on $\partial \mathbb{T}_{r}$, such that for all $x_{1} x_{2} \in S^{(n)}$,

$$
h_{1}^{(n)}\left(x_{1}\right)=\int_{\partial \mathbb{T}_{q}} K_{1}^{(n)}\left(x_{1}, \cdot\right) d \nu_{1}^{(n)} \quad \text { and } \quad h_{2}^{(n)}\left(x_{2}\right)=\int_{\partial \mathbb{T}_{r}} K_{2}^{(n)}\left(x_{2}, \cdot\right) d \nu_{2}^{(n)} .
$$

Since $K_{i}^{(n)}\left(o_{i}, \cdot\right) \equiv 1$, we have $\nu_{1}^{(n)}\left(\partial \mathbb{T}_{q}\right)+\nu_{2}^{(n)}\left(\partial \mathbb{T}_{r}\right)=h\left(o_{1} o_{2}\right)$ for all $n$. Thus, by compactness (Helly's theorem), there are a subsequence $\left(n^{\prime}\right)$ and non-negative measures $\nu_{1}$ on $\partial \mathbb{T}_{q}$ and $\nu_{2}$ on $\partial \mathbb{T}_{r}$ such that $\nu_{i}^{\left(n^{\prime}\right)} \rightarrow \nu_{i}$ weakly for $i=1,2$.

If $x_{1} x_{2} \in D L(q, r)$, then we choose $n_{0}=n_{0}\left(x_{1} x_{2}\right)$ large enough such that for all $n \geq n_{0}$, the geodesics $\overline{O_{i} x_{i}}$ are contained in the interior of $S_{i}^{(n)}, i=1,2$.

For every $\xi_{i} \in \partial \mathbb{T}_{q}$, resp. $\in \partial \mathbb{T}_{r}$, the confluent $c_{i}=x_{i} \wedge \xi_{i}$ is one of the finitely many points on $\overline{o_{i} x_{i}}$. It is clear that $F_{i}^{\partial S_{i}^{(n)}}\left(x_{i}, c_{i}\right) \rightarrow F_{i}\left(x_{i}, c_{i}\right)$ as $n \rightarrow \infty$, and the same is true with $o_{i}$ in the place of $x_{i}$. Therefore, using Lemma 3.7, we find

$$
K_{i}^{(n)}\left(x_{i}, \xi_{i}\right)=K_{i}^{(n)}\left(x_{i}, c_{i}\right) \rightarrow K_{i}\left(x_{i}, c_{i}\right)=K_{i}\left(x_{i}, \xi_{i}\right)
$$


as $n \rightarrow \infty$, where $K_{i}(\cdot, \cdot)$ is the Martin kernel of $P_{i}$ on $\mathbb{T}_{q}$, resp. $\mathbb{T}_{r}, i=1,2$. Thus, $K_{i}^{(n)}\left(x_{i}, \cdot\right) \rightarrow K_{i}\left(x_{i}, \cdot\right)$ uniformly, and using (4.3), we get

$$
h_{1}^{\left(n^{\prime}\right)}\left(x_{1}\right) \rightarrow \int_{\partial \mathbb{T}_{q}} K_{1}\left(x_{1}, \cdot\right) d \nu_{1}=: h_{1}\left(x_{1}\right) \quad \text { and } \quad h_{2}^{\left(n^{\prime}\right)}\left(x_{2}\right) \rightarrow \int_{\partial \mathbb{T}_{r}} K_{1}\left(x_{2}, \cdot\right) d \nu_{2}=: h_{2}\left(x_{2}\right) \text {. }
$$

Then $h_{1}$ is $P_{1}$-harmonic on $\mathbb{T}_{q}$ and $h_{2}$ is $P_{2}$-harmonic on $\mathbb{T}_{r}$, and $h\left(x_{1} x_{2}\right)=h_{1}\left(x_{1}\right)+h_{2}\left(x_{2}\right)$ for all $x_{1} x_{2} \in D L(q, r)$.

We remark that the last part is is the key point of the argument, namely a way of recovering a Poisson integration formula from the finite approximations $K_{i}^{(n)}(\cdot, \cdot)$ of the Martin kernel.

(4.4) Theorem. (a) Each of the functions

$$
x_{1} x_{2} \mapsto K_{1}\left(x_{1}, \xi_{1}\right), \xi_{1} \in \partial^{*} \mathbb{T}_{q}, \quad \text { and } \quad x_{1} x_{2} \mapsto K_{2}\left(x_{2}, \xi_{2}\right), \xi_{2} \in \partial^{*} \mathbb{T}_{r},
$$

is minimal $P_{\alpha}$-harmonic on $D L(p, q)$.

(b) If $\alpha \neq 1 / 2$ then these are all minimal harmonic functions.

(c) If $\alpha=1 / 2$, then these together with the constant function $\mathbf{1}$ are all minimal harmonic functions.

Proof. (a) Let $\xi_{1} \in \partial^{*} \mathbb{T}_{q}$ and suppose that $K_{1}\left(x_{1}, \xi_{1}\right) \geq h\left(x_{1} x_{2}\right)$ for all $x_{1} x_{2} \in D L(q, r)$, where $h \geq 0$ is $P_{\alpha}$-harmonic. By Theorem 2.10, $h\left(x_{1} x_{2}\right)=h_{1}\left(x_{1}\right)+h_{2}\left(x_{2}\right)$, where $h_{i} \geq 0$ is $P_{i}$-harmonic, $i=1,2$. Then $K_{1}\left(\cdot, \xi_{1}\right) \geq h_{1}$. By Proposition 3.8 $h_{1}=c \cdot K_{1}\left(\cdot, \xi_{1}\right)$, where $0 \leq c \leq 1$. If $c=1$ then we are done. Otherwise, $K_{1}\left(x_{1}, \xi_{1}\right) \geq c \cdot K_{1}\left(\cdot, \xi_{1}\right)+h_{2}\left(x_{2}\right)$, that is,

$$
K_{1}\left(x_{1}, \xi_{1}\right) \geq \frac{1}{1-c} h_{2}\left(x_{2}\right)=\int_{\partial T_{r}} K_{2}\left(x_{2}, \cdot\right) d \nu_{2} \quad \forall x_{1} x_{2} \in D L(q, r),
$$

where $\nu_{2}$ is a non-negative Borel measure on $\partial \mathbb{T}^{r}$.

Setting $x_{2}=o_{2}$, we obtain

$$
K_{1}\left(x_{1}, \xi_{1}\right) \geq \nu_{2}\left(\partial \mathbb{T}_{r}\right) \quad \forall x_{1} \in H_{0}\left(\mathbb{T}_{q}\right) .
$$

If $x_{1} \rightarrow \omega_{1}$ then $\mathfrak{h}\left(x_{1}, \xi_{1}\right) \rightarrow \infty$. Therefore, (3.12) yields

$$
\nu_{2}\left(\partial \mathbb{T}_{r}\right) \leq \lim _{x_{1} \rightarrow \omega_{1}, \mathfrak{h}\left(x_{1}\right)=0} K_{1}\left(x_{1}, \xi_{1}\right)=0,
$$

and $\nu_{2}\left(\partial \mathbb{T}_{r}\right)=0$. Therefore $h_{2} \equiv 0$.

This proves minimality of $K_{1}\left(\cdot, \xi_{1}\right)$ for all $\xi_{1} \in \partial^{*} \mathbb{T}_{q}$. Exchanging the roles of the two trees, we get the other "half" of statement (a).

(b,c) Conversely, let $h$ be a minimal $P_{\alpha}$-harmonic function on $D L(q, r)$. By Theorem 2.10. $h\left(x_{1} x_{2}\right)=h_{1}\left(x_{1}\right)+h_{2}\left(x_{2}\right)$ with $h_{i}$ non-negative and $P_{i}$-harmonic. One of the $h_{i}$, say $h_{1}$, must be positive. Minimality yields $h_{1}\left(x_{1}\right)=c_{1} \cdot h\left(x_{1} x_{2}\right)$ for all $x_{1} x_{2} \in D L(q, r)$, where $c_{1}>0$. Thus $h\left(x_{1} x_{2}\right)$ depends only on $x_{1}$. Without loss of generality, $c_{1}=1$, and $h\left(x_{1} x_{2}\right)=h_{1}\left(x_{1}\right)$ for all $x_{1} x_{2}$. Minimality of $h$ with respect to $P_{\alpha}$ yields minimality of $h_{1}$ with respect to $P_{1}$. Thus, by Proposition 3.8 $h\left(x_{1} x_{2}\right)=K_{1}\left(x_{1}, \xi_{1}\right)$ for some $\xi_{1} \in \partial \mathbb{T}_{q}$. 
The case when $h\left(x_{1} x_{2}\right)$ depends only on $x_{2}$ is analogous.

To complete the proof of statments (b) and (c), we have to study minimality of the functions $x_{1} x_{2} \mapsto K_{i}\left(\cdot, \omega_{i}\right), i=1,2$ with respect to $P_{\alpha}$.

If $\alpha=1 / 2$ then by (3.11), $K_{i}\left(\cdot, \omega_{i}\right) \equiv 1$. In this case it is known from KaIMANOviCH AND Woess [12], §6.2, that the Poisson boundary is trivial, i.e., all bounded harmonic functions are constant, which is the same as minimality of the constant function $\mathbf{1}$. This proves (c).

Suppose $\alpha \neq 1 / 2$. Then - again by [12 - the Poisson boundary is nontrivial, and the constant function 1 is non-minimal. If $\alpha>1 / 2$, then this yields that $x_{1} x_{2} \mapsto K_{2}\left(x_{2}, \omega_{2}\right)=$ 1 is non-minimal. On the other hand, we know from (3.11) that

$$
g\left(x_{1} x_{2}\right):=K_{1}\left(x_{1}, \omega_{1}\right)=\left(\frac{1-\alpha}{\alpha}\right)^{\mathfrak{h}\left(x_{1}\right)}
$$

We can conjugate $P_{\alpha}$ by $g$, that is, we set

$$
\check{p}\left(x_{1} x_{2}, y_{1} y_{2}\right)=\frac{p\left(x_{1} x_{2}, y_{1} y_{2}\right) g\left(y_{1} y_{2}\right)}{g\left(x_{1} x_{2}\right)} .
$$

Then $g$ is minimal $P_{\alpha}$-harmonic if and only if $\mathbf{1}$ is minimal $\check{P}_{\alpha}$-harmonic. However, $\check{P}_{\alpha}=P_{1-\alpha}$ by a straightforward computation, and the constant function $\mathbf{1}$ is not minimal $P_{1-\alpha}$-harmonic by non-triviality of the Poisson boundary. Thus, also $g$ is non-minimal for $P_{\alpha}$. Again, the case $\alpha<1 / 2$ follows by exchanging the roles of the two trees.

We remark that for our nearest neighbour case, minimality, resp. non-minimalty of $\mathbf{1}$ can be proved in a more elementary (somewhat longer) way than by appealing to the results of KAIMANOVICH AND WOESS 12.

(4.5) Example. We conclude this section by retranslating the results for simple random walk on $D L(q, q)$ to the setting and notation of the random walk (2.4) on $D L(q, q)$ ("walk forward and switch or switch and walk backward"). We write $\mathbb{T}^{1}$ and $\mathbb{T}^{2}$ for the first and the second tree, respectively. (Both are copies of $\mathbb{T}_{q}$.) We have $\alpha=1 / 2$, and the constant harmonic function $\mathbf{1}$ is minimal.

In terms of configurations, each $\xi_{1} \in \partial^{*} \mathbb{T}^{1}$ corresponds to an infinite configuration

$$
\xi_{1}: \mathbb{Z} \rightarrow \mathbb{Z}_{q} \quad \text { with } \quad \xi_{1}(n)=0 \forall n \leq n_{0}\left(\xi_{1}\right) \in \mathbb{Z} .
$$

If we label the edges of $\mathbb{T}^{1}$ by elements of $\mathbb{Z}_{q}$, as described in (2.1), then $\xi_{1}(n)$ is the label of the edge between the horocycles $H_{n-1}\left(\mathbb{T}^{1}\right)$ and $H_{n}\left(\mathbb{T}^{1}\right)$ on the infinite geodesic $\overline{\omega_{1} \xi_{1}}$. Now let $(\eta, k) \in \mathbb{Z}_{q} \imath \mathbb{Z}$, and consider the associated pair $x_{1} x_{2} \in D L(q, q)$. From the computations in Example 3.9. we know that

$$
K_{1}\left(x_{1}, \xi_{1}\right)=q^{\mathfrak{h}\left(x_{1} \curlywedge \xi_{1}\right)-\mathfrak{h}\left(o_{1} \curlywedge \xi_{1}\right)}
$$


It is easy to compute in terms of $(\eta, k)$

$$
\begin{aligned}
& \mathfrak{h}\left(x_{1} \curlywedge \xi_{1}\right)= \begin{cases}\min \left\{n \leq k: \xi_{1}(n+1) \neq \eta(n+1)\right\} & \text { if such } n \text { exists, } \\
k & \text { otherwise, }\end{cases} \\
& \mathfrak{h}\left(o_{1} \curlywedge \xi_{1}\right)= \begin{cases}\min \left\{m \leq 0: \xi_{1}(m+1) \neq 0\right\} & \text { if such } m \text { exists, } \\
0 & \text { otherwise. }\end{cases}
\end{aligned}
$$

We shall write $\operatorname{def}^{+}\left((\eta, k), \xi_{1}\right)=\mathfrak{h}\left(x_{1} \curlywedge \xi_{1}\right)-\mathfrak{h}\left(o_{1} \curlywedge \xi_{1}\right)$, the (positive) defect of $(\eta, k)$ with respect to $\xi_{1}$.

Analogously, each $\xi_{2} \in \partial^{*} \mathbb{T}^{2}$ corresponds to an infinite configuration

$$
\xi_{2}: \mathbb{Z} \rightarrow \mathbb{Z}_{q} \quad \text { with } \quad \xi_{2}(n)=0 \forall n \geq n_{0}\left(\xi_{2}\right) \in \mathbb{Z}
$$

Here, we label the edges of $\mathbb{T}^{2}$ by elements of $\mathbb{Z}_{q}$, and $\xi_{2}(n)$ is the label of the edge between the horocycles $H_{-n}\left(\mathbb{T}^{2}\right)$ and $H_{-n+1}\left(\mathbb{T}^{2}\right)$ on the infinite geodesic $\overline{\omega_{2} \xi_{2}}$. If $(\eta, k) \in \mathbb{Z}_{q} \imath \mathbb{Z} \leftrightarrow$ $x_{1} x_{2} \in D L(q, q)$, then

$$
K_{1}\left(x_{2}, \xi_{2}\right)=q^{\mathfrak{h}\left(x_{2} \curlywedge \xi_{2}\right)-\mathfrak{h}\left(o_{2} \curlywedge \xi_{2}\right)}
$$

and we compute

$$
\begin{aligned}
& -\mathfrak{h}\left(x_{2} \curlywedge \xi_{2}\right)= \begin{cases}\max \left\{n>k: \xi_{2}(n) \neq \eta(n)\right\} & \text { if such } n \text { exists, } \\
k & \text { otherwise, }\end{cases} \\
& -\mathfrak{h}\left(o_{2} \curlywedge \xi_{2}\right)= \begin{cases}\max \left\{m>0: \xi_{2}(m) \neq 0\right\} & \text { if such } m \text { exists, } \\
0 & \text { otherwise. }\end{cases}
\end{aligned}
$$

We shall write $\operatorname{def}^{-}\left((\eta, k), \xi_{2}\right)=\mathfrak{h}\left(x_{2} \curlywedge \xi_{2}\right)-\mathfrak{h}\left(o_{2} \curlywedge \xi_{2}\right)$, the (negative) defect of $(\eta, k)$ with respect to $\xi_{2}$.

We conclude: write $\partial^{+}\left(\mathbb{Z}_{q} \prec \mathbb{Z}\right)$ and $\partial^{-}\left(\mathbb{Z}_{q} \prec \mathbb{Z}\right)$ for all infinite configurations $\xi_{1}$, resp. $\xi_{2}$ as above. (Every finitely supported configuration appears once in each of the two parts of the boundaries !)

Then all non-constant minimal $P$-harmonic functions are given by

$$
\begin{aligned}
& K_{1}\left(\cdot, \xi_{1}\right)=q^{\operatorname{def}^{+}\left(\cdot, \xi_{1}\right)}, \xi_{1} \in \partial^{+}\left(\mathbb{Z}_{q} \prec \mathbb{Z}\right), \quad \text { and } \\
& K_{2}\left(\cdot, \xi_{2}\right)=q^{\operatorname{def}^{-}\left(\cdot, \xi_{2}\right)}, \xi_{2} \in \partial^{-}\left(\mathbb{Z}_{q} \prec \mathbb{Z}\right) .
\end{aligned}
$$

The constant function $\mathbf{1}$ is also minimal harmonic.

\section{SWITCH-WALK-SWITCH}

We now turn our attention to the random walk (2.6), where at each step, the lamplighter first switches the lamp at his actual position to a random state, then walks, and then switches the lamp at the arrival point to a random state.

As explained in Section 2, this corresponds to simple random walk on the modification of $\operatorname{DL}(q, q)$ where in the first tree, additional edges are drawn between every vertex and the siblings of its predecessor, while the second tree remains as it is. 
More generally, consider $D L(q, r)$. For $x_{1}, u_{1} \in \mathbb{T}_{q}$, we introduce the sibling relation $x_{1} \stackrel{s}{\sim} u_{1} \Longleftrightarrow x_{1}^{-}=u_{1}^{-}$. We extend this relation to $D L(q, r)$ by setting $x_{1} x_{2} \stackrel{s}{\sim} u_{1} x_{2} \Longleftrightarrow$ $x_{1} \stackrel{s}{\sim} u_{1}$. The new ege set on the same vertex set $\left\{x_{1} x_{2} \in \mathbb{T}_{q} \times \mathbb{T}_{r}: \mathfrak{h}\left(x_{1}\right)+\mathfrak{h}\left(x_{2}\right)=0\right\}$ is now given by

$$
\left\{\left[x_{1} x_{2}, y_{1} y_{2}\right]: y_{1}^{-\stackrel{s}{\sim}} x_{1} \text { and } y_{2}=x_{2}^{-}\right\}
$$

We write $D L^{s}(q, r)$ for the resulting graph. Every vertex $x_{1} x_{2}$ with $\mathfrak{h}\left(x_{1}\right)=k$ has $q^{2}$ neighbours $y_{1} y_{2}$ with $\mathfrak{h}\left(y_{1}\right)=k+1$ and $q r$ neighbours with $\mathfrak{h}\left(y_{1}\right)=k-1$. Adapted to this structure, we choose $0<\alpha<1$ and consider the random walk on $D L^{s}(q, r)$ with transition matrix $Q=Q_{\alpha}$ given by

$$
q\left(x_{1} x_{2}, y_{1} y_{2}\right)= \begin{cases}\alpha / q^{2} & \text { if } y_{1}^{-} \stackrel{s}{\sim} x_{1} \text { and } y_{2}=x_{2}^{-} \\ (1-\alpha) /(q r) & \text { if } y_{1} \stackrel{s}{\sim} x_{1}^{-} \text {and } y_{2}^{-}=x_{2} \\ 0 & \text { otherwise }\end{cases}
$$

Now note that when $x_{1} \stackrel{s}{\sim} u_{1}$ then transitions from $x_{1} x_{2}$ and $u_{1} x_{2}$ go to the same neighbours with the same probabilities. Thus, $Q h\left(x_{1} x_{2}\right)=Q h\left(u_{1} x_{2}\right)$ whenever $x_{1} \stackrel{s}{\sim} u_{1}$, and we have the following.

(5.2) Lemma. Every $Q_{\alpha}$-harmonic function is constant on each equivalence class of the sibling relation.

We can construct the factor graph of $D L^{s}(q, r)$ with respect to the sibling relation. We write $\left[x_{1}\right] x_{2}$ for the equivalence class of $x_{1} x_{2}$, since all its elements have the same second "coordinate", and $\left[x_{1}\right]$ is the sibling class in the first tree. Then the vertex set of the factor graph is $\left\{\left[x_{1}\right] x_{2}: x_{1} x_{2} \in D L^{s}(q, r)\right\}$, and two classes $\left[x_{1}\right] x_{2}$ and $\left[y_{1}\right] y_{2}$ are connected by an edge of the factor graph if and only if there is an edge bewteen a pair of representatives in $D L^{s}(q, r)$. Thus, if $\mathfrak{h}\left(y_{2}\right)=\mathfrak{h}\left(x_{2}\right)-1$, there is an edge from $\left[x_{1}\right] x_{2}$ to $\left[y_{1}\right] y_{2}$ precisely when $\left[y_{1}^{-}\right]=\left[x_{1}\right]$ and $x_{2}^{-}=y_{2}$. We write $\pi$ for the natural projection. The next lemma is now immediate.

(5.3) Lemma. The factor graph of $D L^{s}(q, r)$ with respect to the sibling relation is $D L(q, r)$.

The transition matrix $Q_{\alpha}$ is compatible with the factorization, and its image under the projection $\pi: D L^{s}(q, r) \rightarrow D L(q, r)$ is $P_{\alpha}$, as defined in 2.7).

By "compatible" we mean that $q_{\alpha}\left(v_{1} x_{2},\left[y_{1}\right] y_{2}\right)=\sum_{w_{1}} \stackrel{s}{\sim} y_{1} q_{\alpha}\left(x_{1} x_{2}, w_{1} y_{2}\right)$ is the same for each representative $v_{1} \in\left[x_{1}\right]$, and this common value is the transition probability from $\left[x_{1}\right] x_{2}$ to $\left[y_{1}\right] y_{2}$ of the projection of $Q_{\alpha}$.

(5.4) Corollary. Every $Q_{\alpha}$-harmonic function is of the form $h \circ \pi$, where $h$ is a $P_{\alpha^{-}}$ harmonic function on $D L(q, r)$ and $\pi: D L^{s}(q, r) \rightarrow D L(q, r)$ is the factor map with respect to the sibling relation.

(5.5) Example. Our final task is to retranslate once more to the lamplighter group, by giving a direct description of the minimal harmonic functions for the switch-walk-switch model that does not involve the above factor map. We have $Q=Q_{1 / 2}$ on $D L^{s}(q, q)$. 
Now, it is clear what the factor map does to a pair $(\eta, k) \in \mathbb{Z}_{q} \prec \mathbb{Z}$ : it "forgets" (cancels) the value $\eta(k)$, and what remains is the pair $\left(\eta_{k}, k\right)$, where

$$
\eta_{k}(n)= \begin{cases}\eta(n-1) & \text { if } n \leq k, \\ \eta(n) & \text { if } n>k .\end{cases}
$$

Thus, with respect to the computations of Example 4.5, (4.7) remains unchanged, while instead of the positive defect we need

$$
\begin{aligned}
& \operatorname{def}^{\oplus}\left((\eta, k), \xi_{1}\right)=\mathfrak{h}\left(\left[x_{1}\right] \curlywedge \xi_{1}\right)-\mathfrak{h}\left(\left[o_{1}\right] \curlywedge \xi_{1}\right), \quad \text { where } \\
& \mathfrak{h}\left(\left[x_{1}\right] \curlywedge \xi_{1}\right)= \begin{cases}\min \left\{n \leq k: \xi_{1}(n) \neq \eta(n)\right\} & \text { if such } n \text { exists, } \\
k & \text { otherwise }\end{cases} \\
& \mathfrak{h}\left(\left[o_{1}\right] \curlywedge \xi_{1}\right)= \begin{cases}\min \left\{m \leq 0: \xi_{1}(m) \neq 0\right\} & \text { if such } m \text { exists } \\
0 & \text { otherwise. }\end{cases}
\end{aligned}
$$

Again, the constant function $\mathbf{1}$ is minimal $Q$-harmonic. All non-constant minimal $Q$ harmonic functions are given by

$$
\begin{aligned}
& K_{1}\left(\cdot, \xi_{1}\right)=q^{\operatorname{def}^{\oplus}\left(\cdot, \xi_{1}\right)}, \xi_{1} \in \partial^{+}\left(\mathbb{Z}_{q} \prec \mathbb{Z}\right), \quad \text { and } \\
& K_{2}\left(\cdot, \xi_{2}\right)=q^{\operatorname{def}^{-}\left(\cdot, \xi_{2}\right)}, \xi_{2} \in \partial^{-}\left(\mathbb{Z}_{q} \prec \mathbb{Z}\right) .
\end{aligned}
$$

\section{Final observations AND SPECUlations}

(6.1) Even when $r \neq q$, one can interpret $D L(q, r)$ as a "lamplighter graph" over $\mathbb{Z}$ : at each point of $\mathbb{Z}$, there are green lamps with $q$ different states, including "off", and red lamps with $r$ different states, again including "off". The lamplighter walking along $\mathbb{Z}$ has to make sure that when his actual position is $k \in \mathbb{Z}$, then the lamps in $(-\infty, k]$ have to be in one of the green states, and those in $[k+1,+\infty)$ in one of the red states.

(6.2) Several basic properties of random walks on $D L(q, r)$ that are not necessarily of nearest neighbour type, but invariant under a transitive group of automorphisms of $D L(q, r)$, were studied by BERTACCHI [1]. For a large class of random walks of this type, the Poisson boundary was determined by KAIMANOVICH AND WoEss [12, as mentioned above.

(6.3) As a general principle, the three problems of (i) determining the Poisson boundary (三 all bounded harmonic functions), (ii) determining all minimal harmonic functions (三 all positive harmonic functions), and (iii) determining the full Martin compactification (三 finding the directions of convergence of the Martin kernels) have an increasing degree of difficulty. (As a matter of fact, these three problems get sometimes mixed up even by advanced non-experts.) Thus, the reader should not be a priori astonished by the fact that in this paper, we were able to solve problem (ii) for a much smaller class of random walks than those for which (i) was solved in [12]. 
(6.4) In particular, to the author's knowledge, the present results provide the first example of a complete solution of problem (ii) on a finitely generated, solvable group. In addition, our group is non-polycyclic.

On the other hand, the situation is much better understood for connected solvable Lie groups, because more structure theory is at hand. For the basic example, namely the affine group over $\mathbb{R}$, random walks and harmonic functions were studied in much detail, see Molchanov [14, Elie [7], or Bougerol And Elie [2]. We recall here that the main result of [2] implies existence of non-constant positive harmonic functions for finite range random walks on finitely generated polycyclic groups with exponential growth, but a complete solution of (ii) is not available for those groups.

As for the affine group over $\mathbb{R}$, the Poisson boundary and the Martin compactification are well understood for random walks on the affine group over the $p$-adic numbers, see Cartwright, Kaimanovich and Woess [5] and Brofferio [3].

(6.5) As pointed out by BERTACCHI [1, there is a natural geometric compactification of $D L(q, r)$. Namely, this graph is a subgraph of the direct product $\mathbb{T}_{q} \times \mathbb{T}_{r}$, for which a natural compactification is $\widehat{\mathbb{T}}_{q} \times \widehat{\mathbb{T}}_{r}$. Thus, we define $\widehat{D L}(q, r)$ as the closure of $D L(q, r)$ in $\widehat{\mathbb{T}}_{q} \times \widehat{\mathbb{T}}_{r}$, and $\partial D L(q, r)=\widehat{D L}(q, r) \backslash D L(q, r)$. Almost sure convergence of random walks to a boundary-valued random variable is studied in [1]. However, at present, we are far from proving that this compactification is in some sense comparable or identical with the Martin compactification even in the case of simple random walk.

(6.6) The following seems noteworthy regarding the two classes of examples that we have studied: in the case of drift $(\alpha \neq 1 / 2)$, the minimal harmonic functions are parametrized (continuously on each part) by $\partial^{*} \mathbb{T}_{q} \cup \partial^{*} \mathbb{T}_{r}$. In the drift-free case $(\alpha=1 / 2)$, the additional minimal harmonic function 1 enters the stage. Thus, in some sense, the cone of positive harmonic functions is bigger in the driftfree case than in presence of drift. This contrasts with all examples known so far. (Of course, the constant function $\mathbf{1}$ is a Martin kernel even when $\alpha \neq 1 / 2$, but then it does not belong to $\mathcal{M}_{\min }$.)

(6.7) Finally, the reason why our method does not adapt to the "walk or switch" model (2.5) appears to be that in contrast with the cases that we have solved here, this random walk is not invariant under a group of automorphisms of $D L(q, r)$ that acts transitively both on $\partial^{* *} \mathbb{T}^{1}$ and $\partial^{*} \mathbb{T}^{2}$.

A next step could be to try to prove the Decomposition Theorem 2.10 for all irreducible random walks with the latter transitivity property.

Acknowledgement. The author is grateful to Rögnvaldur I. Möller for pointing out the relationship between the Diestel-Leader graphs $D L(q, q)$ and the lamplighter groups.

\section{REFERENCES}

[1] Bertacchi, D.: Random walks on Diestel-Leader graphs, Abh. Math. Sem. Univ. Hamburg 71 (2001) 205-224.

[2] Bougerol, Ph., and Elie, L.: Existence of non-negative harmonic functions on groups and on covering manifolds, Ann. Inst. Poincaré (Probab. Stat.) 31 (1995) 59-80. 
[3] Brofferio, S.: Marches aléatories sur les groupes affines de l'arbre et de la droite réelle et processus localment contractifs, Thèse, Univ. Paris 6 (2002).

[4] Cartier, P.: Fonctions harmoniques sur un arbre, Symposia Math. 9 (1972) 203-270.

[5] Cartwright, D. I., Kaimanovich, V. A., and Woess, W.: Random walks on the affine group of local fields and of homogeneous trees, Ann. Inst Fourier (Grenoble) 44 (1994) 1243-1288.

[6] Diestel, R., and Leader, I.: A conjecture concerning a limit of non-Cayley graphs, J. Algebraic Combin. 14 (2001) 17-25.

[7] Elie, L.: Fonctions harmoniques positives sur le groupe affine, in: Probability Measures on Groups, (ed. H. Heyer.) pp. 96-110, Lect. Notes in Math. 706, Springer, Berlin, 1978.

[8] Erschler, A. G.: On the asymptotics of the rate of departure to infinity (Russian), Zap. Nauchn. Sem. S.-Peterburg. Otdel. Mat. Inst. Steklov. (POMI) 283 (2001) 251-257, 263.

[9] Grigorchuk, R. I., and Żuk, A.: The lamplighter group as a group generated by a 2-state automaton, and its spectrum, Geom. Dedicata 87 (2001) 209-244.

[10] Kaimanovich, V. A.: Poisson boundaries of random walks on discrete solvable groups, in: Probability Measures on Groups X (ed. H. Heyer), pp. 205-238, Plenum, New York, 1991.

[11] Kaimanovich, V. A., and Vershik, A. M.: Random walks on discrete groups: boundary and entropy, Ann. Probab. 11 (1983) 457-490.

[12] Kaimanovich, V. A., and Woess, W.: Boundary and entropy of space homogeneous Markov chains, Ann. Probab. 30 (2002) 323-363.

[13] Lyons, R., Pemantle, R., and Peres, Y.: Random walks on the lamplighter group, Ann. Probab. 24 (1996) 1993-2006.

[14] Molchanov, S. A.: Martin boundaries for invariant Markov processes on a solvable group, Theory of Probability and Appl. 12 (1967) 310-314.

[15] Picardello, M. A., Taibleson, M. H., and Woess, W.: Harmonic functions on Cartesian products of trees with finite graphs, J. Functional Analysis 102 (1991) 379-400.

[16] Pittet, C., and Saloff-Coste, L.: Amenable groups, isoperimetric profiles and random walks, in: Geometric Group Theory Down Under (Canberra, 1996), pp. 293-316, de Gruyter, Berlin, 1999.

[17] Pittet, C., and Saloff-Coste, L.: On random walks on wreath products, Ann. Probab. 30 (2002) 948-977.

[18] Revelle, D.: Rate of escape of random walks on wreath products, Ann. Probab. 31 (2003), to appear.

[19] Revelle, D.: Heat kernel asymptotics on the lamplighter group, preprint, Cornell Univ. (2002).

[20] Woess, W.: Random Walks on Infinite Graphs and Groups, Cambridge Tracts in Mathematics 138, Cambridge University Press, Cambridge, 2000.

E-mail address: woess@TUGraz.at

Institut Für Mathematik C, Technische Universität GraZ

Steyrergasse 30, A-8010 Graz, Austria 